\title{
La rotación de puestos de trabajo como medio para la integración de trabajadores con discapacidad
}

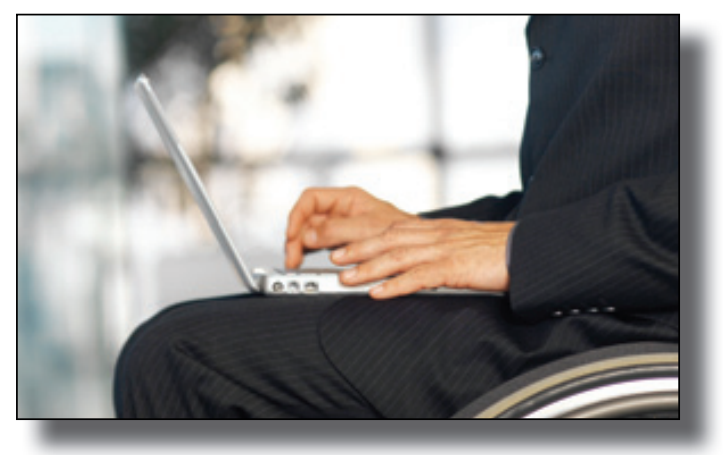

\author{
Sabina Asensio-Cuesta* \\ Dra. Ingeniera Informática \\ José Antonio Diego-Mas* \\ María Carmen González-Cruz* \\ Dr. Ingeniero Industrial \\ Dra. Ingeniera Industrial
}
* UNIVERSIDAD POLITÉCNICA DE VALENCIA. Dpto. de Proyectos de Ingeniería. Camino de Vera, $s / n$ - 46020 Valencia. Tfno: +34963 877007. sasensio@dpi.upv.es; jodiemas@dpi.upv.es; megonzal@dpi.upv.es

\section{Job rotation as a method for disabled workers integration}

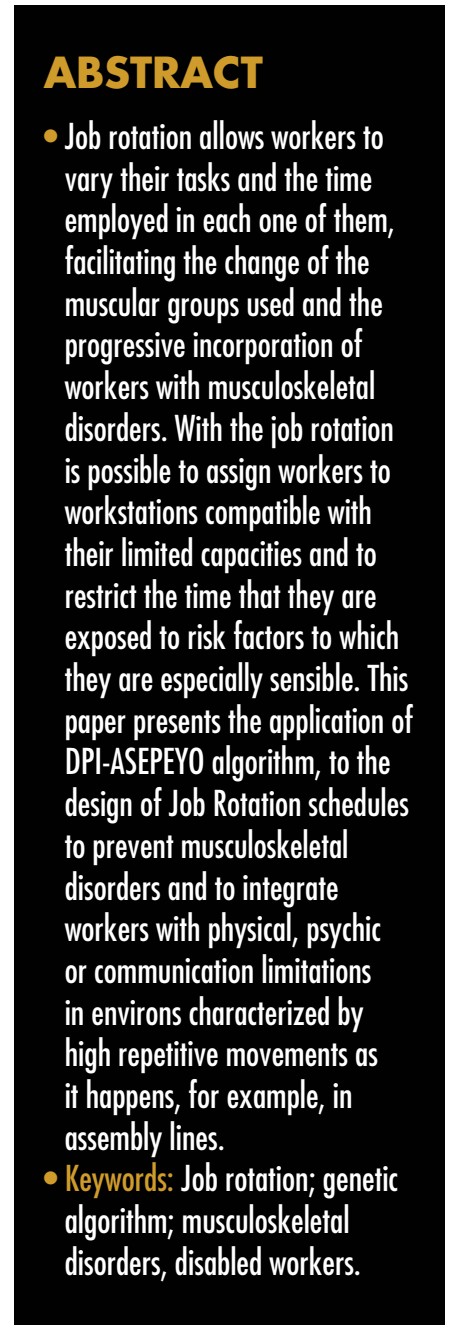

\section{RESUMEN}

La Rotación de Puestos de Trabajo permite la variación de las tareas llevadas a cabo por los trabajadores y el tiempo empleado en cada una de ellas, facilitando la alternancia de los grupos musculares utilizados y la incorporación progresiva de trabajadores con problemas músculoesqueléticos. Con la rotación es posible asignar a los trabajadores limitados a puestos compatibles con sus capacidades y restringir el tiempo que se exponen a factores de riesgo a los que son especialmente sensibles.

Este trabajo presenta la aplicación del algoritmo DPI-ASEPEYO al diseño de agendas de RPT para prevenir los trastornos músculo-esqueléticos e integrar a trabajadores con limitaciones físicas, psíquicas o de comunicación en entornos de trabajo caracterizados por una elevada repetitividad de movimientos, como ocurre, por ejemplo, en las líneas de ensamblaje.

Palabras clave: rotación de puestos, algoritmos genéticos, trastornos musculo-esquelético, trabajadores discapacitados.

\section{INTRODUCCIÓN}

Cada vez más, las empresas son conscientes de su responsabilidad en la protección de la salud y seguridad de los trabajadores. Dicha actitud resulta beneficiosa para éstas y para la sociedad a nivel socio-económico, al tiempo que repercute positivamente en su reputación e imagen corporativa (Armengou-Foix, 2006; Lopez-Lasaola, 2008).

La Ley Orgánica 13/1982, de 7 de abril, de Integración Social de los Minusválidos (LISMI), señala que la finalidad primordial de la política de empleo de trabajadores con discapacidad debe ser su integración en condiciones que garanticen la aplicación del principio de igualdad de trato, en el sistema ordinario de trabajo o, en su defecto, su incorporación al sistema productivo a través de Centros Especiales de Empleo (CEE). Por otra parte, la LISMI indica que las empresas públicas y privadas que empleen a un número de 50 o más trabajadores están obligadas a que, al menos, el $2 \%$ sean trabajadores discapacitados. Sin embargo, todavía son numerosas las empresas que no cubren dicho cupo (Oromí, 2006).

Según la "Encuesta de Discapacidad, Autonomía personal y situaciones 
de Dependencia" publicada por el Instituto Nacional de Estadística (España) en 2008, las deficiencias más frecuentes entre los discapacitados se localizan en las articulaciones y los huesos, que afectan a más de 1,2 millones de ellos. Dichas discapacidades pertenecen al grupo de dolencias denominadas trastornos músculo-esqueléticos (TMEs). Por otra parte, el mayor porcentaje de personas con discapacidades ocupadas, se presentaba en las personas con discapacidades auditivas y visuales $(42,8 \%$ y $32,8 \%$ respectivamente), seguida de aquellos con problemas de movilidad (21,6\%). Resulta significativo que en 2008 , de las personas discapacitadas ocupadas, 711.700 tuvieron que cambiar de trabajo o dejar de trabajar por su discapacidad.

Todo esto hace necesario ofrecer a las empresas mecanismos que les facilite cumplir con el cupo de ocupación de discapacitados exigido por la legislación vigente. Dicha ocupación debe tener carácter permanente, procurando que los puestos ocupados por los trabajadores con discapacidades sean compatibles con sus limitaciones tanto a corto como a largo plazo. Uno de estos mecanismos puede ser la Rotación de Puestos de Trabajo (RPT) (Vézina, 2004; Costa et al., 2009).

La RPT consiste en el cambio de un operario entre dos o más puestos de trabajo del mismo nivel y contenido tecnológico y/o funcional en periodos regulares o irregulares de tiempo. Es un tipo de organización del trabajo cada vez más extendida entre las empresas manufactureras y de servicios. La RPT puede facilitar tanto el retorno progresivo de trabajadores en proceso de rehabilitación de TMEs, como la integración de trabajadores con otros tipos de discapacidades. Además puede actuar como mecanismo de prevención de los TMEs (Vézina, 2004). La RPT permite asignar a los trabajadores a puestos compatibles con sus limitaciones controlando el tiempo que se exponen a factores de riesgo especialmente dañinos para ellos.

El diseño de una agenda de rotación que considere la integración en las empresas de trabajadores discapacitados es un problema complejo. La empresa debe generar perfiles de competencias para cada puesto y para cada trabajador. De esta forma los trabajadores pueden ser asignados a puestos que requieran las competencias que poseen. Se evita así que la selección de los trabajadores para ocupar los puestos se realice considerando factores sin relación con el adecuado desempeño de las funciones requeridas en ellos. Asimismo, se logra la alineación entre las estrategias organizacionales de la empresa y el desempeño efectivo del trabajador (Millas, 2005).

Por otra parte, para lograr el efecto preventivo de la RPT sobre los TMEs, ésta debe introducir alternancia efectiva entre los grupos musculares empleados por los trabajadores. Dicha técnica también permite reducir la monotonía y el aburrimiento, para lo cual, es necesario que la rotación introduzca variedad en el tipo de actividad mental desarrollada por los trabajadores. Por último, para que sea viable la RPT, se deben tener presentes las restricciones productivas y organizativas presentes en cada empresa, tales como: los periodos de descanso, los tiempos de ciclo o las diferencias salariales entre los trabajadores.

Debido al gran número de aspectos que es necesario considerar a la hora de diseñar una agenda de RPT, el problema planteado pertenece al grupo denominado "Problemas de Horarios y Secuenciación" (Timetabling y Sheduling problems) (Papadimitriou y Steiglitz, 1982). Dichos problemas consisten en localizar la mejor configuración de un grupo de variables que, cumpliendo ciertas restricciones, minimicen (o en su caso maximicen) una función objetivo. Para la obtención de agendas de RPT se ha aplicado la programación entera (Carnahan et al., 2000; Tharmmaphornphilas et al.,2004) y se han propuesto diversas técnicas metaheurísticas (Carnahan et al., 2000; Seçkiner et al., 2007; Costa et al., 2009; Diego-mas et. al., 2009).

En este trabajo se describe la aplicación del algoritmo genético DPI-ASEPEYO (DPI-A) (Diego-mas et. al. ,2009), a un caso de estudio localizado en una línea de ensamblaje. Se desea probar la capacidad del algoritmo para diseñar agendas de rotación que permitan la integración de trabajadores con discapacidades físicas, psíquicas y/o de comunicación en el sistema ordinario de trabajo, al tiempo que contribuyen a prevenir los TMEs.

El contenido del trabajo se estructura como sigue. En la Sección 2 se describe brevemente el algoritmo DPI-A. En la Sección 3 se plantea el caso de estudio. En la Sección 4 se recopilan los datos y parámetros derivados del análisis del caso. En la Sección 5 se muestran los resultados obtenidos al aplicar el algoritmo DPI-A al problema planteado. Finalmente, en la Sección 5, se exponen las conclusiones derivadas del estudio.

\section{DESCRIPCIÓN DEL ALGORITMO DPI-ASEPEYO}

El procedimiento de obtención de agendas de rotación mediante el algoritmo DPI-A se inicia elaborando perfiles competenciales para cada puesto y para cada trabajador. Es decir, estableciendo los requerimientos de los puestos y las limitaciones de los trabajadores. Dichos perfiles se elaboran en base a 18 criterios que representan movimientos (ítems de movimiento) (ver columna 1 de la Tabla 1), y a un conjunto de 17 capacidades globales, psíquicas y de comunicación (ver columna 1 de la Tabla 2).

Los ítems de movimiento son puntuados para los puestos en función de la frecuencia con la que se realiza en ellos el movimiento que representan. Las puntuaciones de los ítems de movimiento tomarán los valores: 3 si el movimiento se realiza más de 8 veces por minuto, 2 si la frecuencia está entre 3 y 7 movimientos por minuto, 1 si se exige un movimiento por minuto y 0 si no se requiere el movimiento en el puesto.

Los trabajadores son valorados para los ítems de movimiento según sus limitaciones para realizar los diferentes movimientos. El algoritmo DPI-A asigna a los trabajadores sin problemas de movilidad un 0 , si presentan limitación baja un 1, si la limitación es alta les asigna un 2 , 
y si son incapaces de realizar el movimiento los puntúa con un 3.

A continuación, se determina el conjunto de capacidades globales, psíquicas y de comunicación que son necesarias en los puestos y se registra qué trabajadores las tienen limitadas.

Obtenidos los perfiles competenciales de los puestos, el algoritmo DPI-A permite obtener agendas de rotación que maximicen la idoneidad de las asignaciones de los trabajadores a los puestos al tiempo que promueven la alternancia entre los grupos musculares empleados.

El algoritmo DPI-A es un algoritmo genético en el que las agendas de rotación se codifican mediante matrices de tamaño $\mathrm{n}_{\text {wor }} \times \mathrm{n}_{\text {rot }}$, siendo $\mathrm{n}_{\text {wor }}$ el número de trabajadores participantes, $\mathrm{y}_{\mathrm{rot}}$ el número de rotaciones. En cada celda se indica el puesto que ocupa un trabajador w en una rotación r. El algoritmo comienza con la generación aleatoria de una población de $n_{i}$ individuos o agendas de rotación factibles, es decir, que no repiten un mismo puesto en una misma rotación. Posteriormente, se evalúa la calidad o aptitud de cada solución. La evaluación de los individuos se realiza en dos fases. En una primera fase se recalculan los valores de los ítems de movimiento de los trabajadores para cada rotación para reflejar la acumulación de fatiga debido a los puestos ocupados en las rotaciones precedentes (Ecuación 1).

En la siguiente fase se evalúa la aptitud (E) o fitness de cada individuo o agenda de rotación (Ecuación 2). Para ello se emplean los valores de los ítems de movimientos recalculados en la fase anterior y los de los puestos recopilados inicialmente. La aptitud de una agenda dependerá del grado de adecuación de las asignaciones de los trabajadores a los puestos en las diferentes rotaciones.

Una vez obtenida la aptitud, se penalizan, incrementando el valor de E, las agendas con asignaciones no deseadas, como por ejemplo, en las que un trabajador ocupa un puesto que exige capacidades que tiene limitadas, o asignaciones de un trabajador a un puesto en el que son necesarios movimientos que éste no puede realizar. También se penaliza la ocupación consecutiva de un puesto durante cierto tiempo máximo $\left(\mathrm{t}_{\max }\right)$. Finalmente, se seleccionan las soluciones supervivientes que pasarán a la siguiente generación mediante el "Método de la ruleta" (Goldberg, 1989). En dicha selección tendrán más probabilidad de ser elegidos los individuos con menor valor de E, y por tanto, será menos probable que sobrevivan las agendas penalizadas. Con el mismo procedimiento se escogen $n_{i} \bullet p_{c}\left(p_{c}\right.$ probabilidad de cruce) individuos sobre los que actúa el operador cruce. Como resultado del cruce se obtienen $n_{i} \bullet p_{c}$ individuos hijos que pasarán a formar parte de la siguiente generación. Sobre $n_{i} \bullet p_{m}$ individuos, de entre los individuos supervivientes y los individuos hijos, podrá actuar el operador mutación, siendo $\mathrm{p}_{\mathrm{m}}$ la probabilidad de mutación. El algoritmo finaliza trascurrido un número determinado de generaciones. Tras dicho número de generaciones DPI-A propone una agenda de RPT basada en los perfiles

$$
w_{j}^{x}(r)=w^{x}+\frac{1}{f_{\text {red }}} * \sum_{\substack{k=1 \\ p_{i}\left(a^{x}(k)\right)>\text { tre }}}^{r-1} \frac{p_{j}\left(a^{x}(k)\right)^{*} t_{k}}{d_{k}}
$$

Donde:

$$
d_{k}=\left\{\begin{array}{c}
p_{k, r}+\sum_{\substack{g=k+1 \\
1 \quad ; k=r-1}}^{r-1} t_{g} \quad ; k<r-1 \\
\text {; } k=r-1
\end{array}\right.
$$

$d_{k}$ es el tiempo desde el fin de la rotación k y el comienzo de la actual rotación $r_{,}$incluyendo pausas entre ellas $p_{k}$.

$w^{x}{ }_{j}(r)$ es el valor del ítem j para el trabajador $\mathrm{x}$ en la rotación $\mathrm{r}$.

$w^{x}$ es el valor original del ítem j para el trabajador $\mathrm{x}$.

$a^{x}(k)$ es el puesto asignado al trabajador $x$ en la rotación $k$.

$p_{j}\left(a^{*}(k)\right.$ es el valor del ítem jen el puesto asignado al trabajador x en la rotación $k$.

tre es el valor umbral (threshold) de los ítems de los puestos por debajo del cual se considera

que no provocan fatiga.

$t_{k}$ es la duración de la rotación $\mathrm{k}$.

$f_{\text {red }}$ es el factor de reducción o importancia del historial previo de rotaciones.

$p_{k y}$ duración de las pausas entre la rotación $k$ y la rotación $r$.

Ecuación 1: Cálculo del ítem i de un trabajador x para la rotación r 


$$
E=\sum_{x=1}^{n_{\text {resp }}} \sum_{r=1}^{n_{\text {rot }}} \sum_{j=1}^{n_{\text {at }}}\left(C_{j} * w_{j}^{x}(r) * p_{j}\left(\mathrm{a}^{\mathrm{x}}(\mathrm{r})\right) * t_{r}\right)^{u}
$$

\section{Donde:}

$n_{\text {wor }}$ es el número de trabajadores.

$n_{\text {rot }}$ es el número de rotaciones.

$n_{i t}$ número de ítems de movimiento.

$C_{j}$ es el coeficiente de importancia relativa del ítem j respecto al resto de ítems

$w^{x}{ }_{j}(r)$ es el valor del ítem j para el trabajador $\mathrm{x}$ en la rotación $\mathrm{r}$.

$a^{x}(k)$ es el puesto asignado al trabajador $\mathrm{x}$ en la rotación $\mathrm{k}$.

$p_{j}\left(a^{x}(k)\right.$ es el valor del ítem j en el puesto asignado al trabajador $x$ en la rotación $k$.

$t_{r}$ es la duración de la rołación r.

$u$ es el exponente de uniformidad que evita asignaciones descompensadas.

Ecuación 2: Función de evaluación de la aptitud o fitness (E) de una solución o agenda de rotación

competenciales de los puestos y de los trabajadores, y con alternancia de los grupos musculares empleados en cada rotación. En Diego-mas et. al (2009) se describe con detalle el algoritmo DPI-A.
Para la aplicación del algoritmo DPI-A resulta útil el software "MORE" (http://www.ergonautas.upv.es/metodos/ more/more.htm) desarrollado por los propios autores de éste trabajo en colaboración con la mutua de accidentes de trabajo y enfermedades profesionales ASEPEYO.

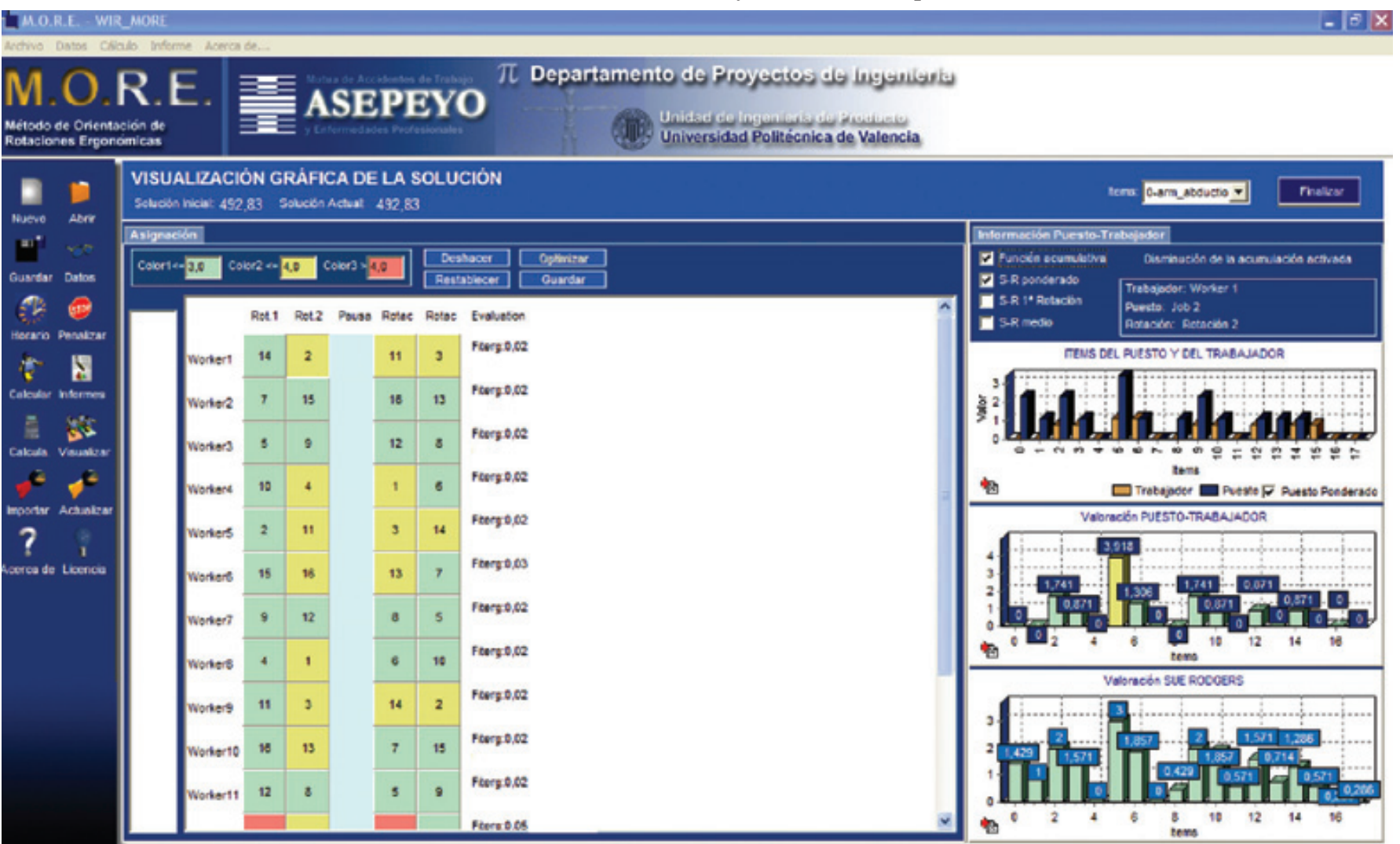

Figura 1: Interfaz principal de la aplicación informática MORE 


\section{DESCRIPCIÓN DEL CASO DE ESTUDIO}

En una línea de ensamblaje se deseaba diseñar un plan de rotaciones para 18 puestos de trabajo y el mismo número de trabajadores. Tres de los trabajadores seleccionados para la rotación estaban en proceso de recuperación de una lesión músculo-esquelética leve, por lo que el equipo médico desaconsejaba su exposición prolongada a determinados movimientos. Asimismo, tenían limitaciones en ciertas capacidades derivadas de sus problemas de movilidad. Además, se quería incorporar a un trabajador con problemas de visión a distancia. La jornada laboral era de 8 horas, con una hora de descanso para el almuerzo. Se establecieron 4 rotaciones, las tres primeras de 2 horas y la última de 1 hora. La pausa de descanso fue situada después de la segunda rotación. La adopción del plan de rotaciones contó con el apoyo de la dirección, y fue enmarcado dentro de un Plan General de Mejora Ergonómica y de Responsabilidad Social Corporativa de la empresa.

\subsection{ESTUDIO DE LA REPETITIVIDAD DE MOVIMIENTOS DE LOS PUESTOS}

La mayoría de los puestos presentaban una elevada repetitividad de movimientos del cuello y de los miembros superiores. Dicha circunstancia complicaba la integración de trabajadores con dificultades de movilidad en el cuello, como por ejemplo, con problemas cervicales. Respecto a los miembros superiores, sólo en el 5,6\% de los puestos era poco frecuente el movimiento de flexión de brazo. En todos los puestos se exigía flexión de antebrazo. Así pues, se hacía difícil la integración de trabajadores con problemas en la flexión de brazo o antebrazo (epicondilitis o tendinitis).
Respecto al movimiento de elevación del hombro se observó que en la mayoría de los puestos era poco frecuente y en el resto inexistente. En el caso de los movimientos que afectaban a la muñeca y a los dedos, en todos los puestos se exigía flexión de muñeca, en la mayoría de los casos con frecuencia moderada o baja. La extensión de muñeca, o bien no existía, o en los puestos en los que estaba presente se exigía a baja frecuencia. El giro de muñeca se observó en todos los puestos. Por otra parte, el movimiento de lateralización no se realizaba en todos los puestos, y en los casos en los que era necesario, se realizaba a baja frecuencia. Todos los puestos requerían el empleo de los dedos para realizar agarres, en la mayor parte de los puestos a frecuencia moderada. Dichos resultados ponían de manifiesto la dificultad para integrar en la línea a trabajadores con lesiones en la muñeca o en los dedos, como por ejemplo, trabajadores con el síndrome del túnel carpiano o dedo en gatillo.

El movimiento de flexión de tronco se exigía en todos los puestos. En ningún puesto era necesaria la extensión de tronco. La rotación del tronco tenía una repetitividad moderada o poco frecuente. La lateralización de tronco exigida en los puestos era poco frecuente o inexistente. Así pues resultaba complicada la inclusión de trabajadores con problemas en la flexión del tronco, no así en los movimientos de extensión y lateralización de dicho miembro.

Finalmente, la flexión de piernas no se exigía en todos los puestos, $y$, en los que estaba presente, la frecuencia de la repetición era moderada o poco frecuente. La escasa frecuencia de flexión de piernas podía facilitar el trabajo de operarios con problemas leves en dicho movimiento.

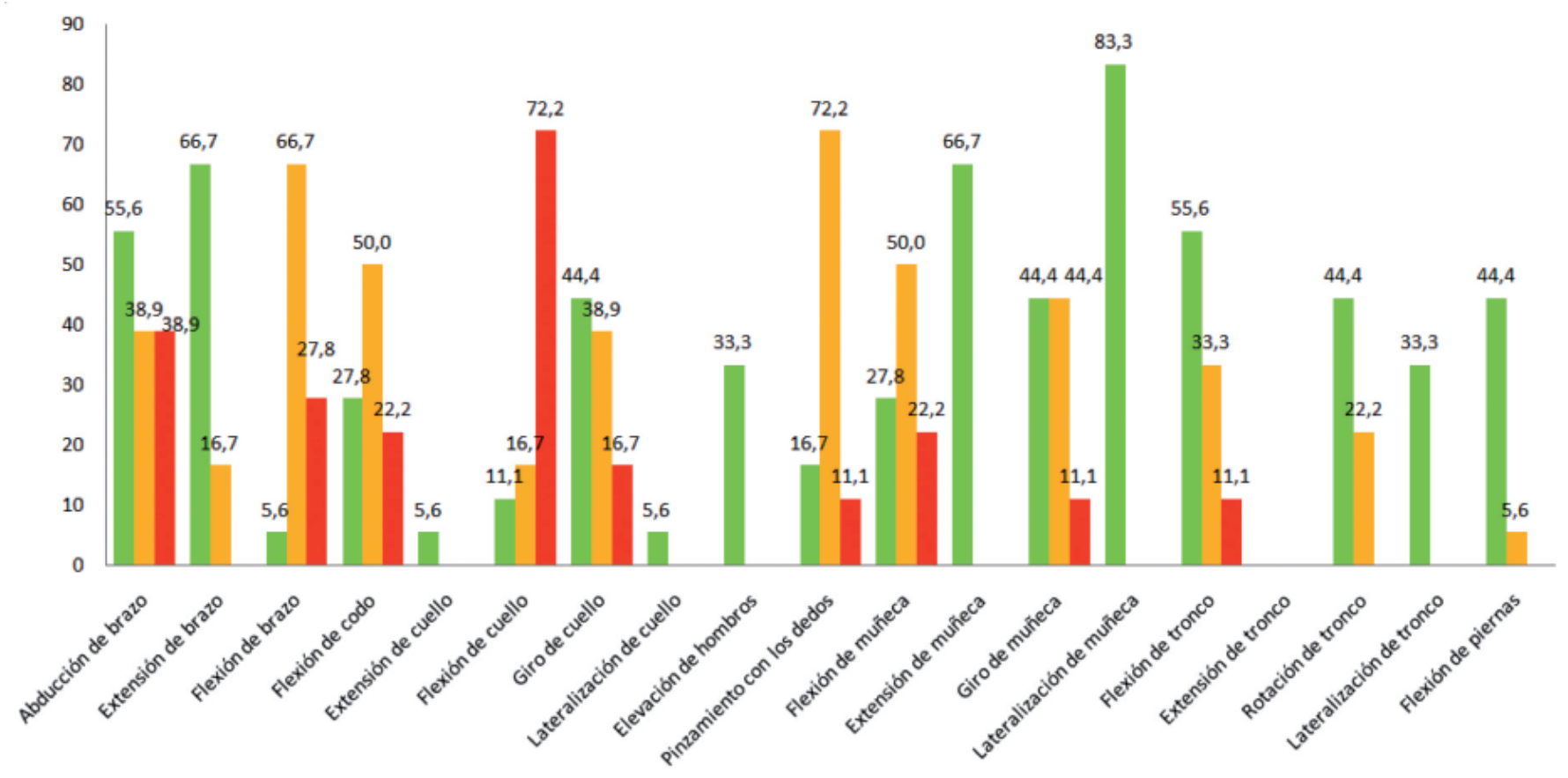

Fig. 2: Porcentajes de frecuencias de los 19 movimientos analizados en los 18 puestos 


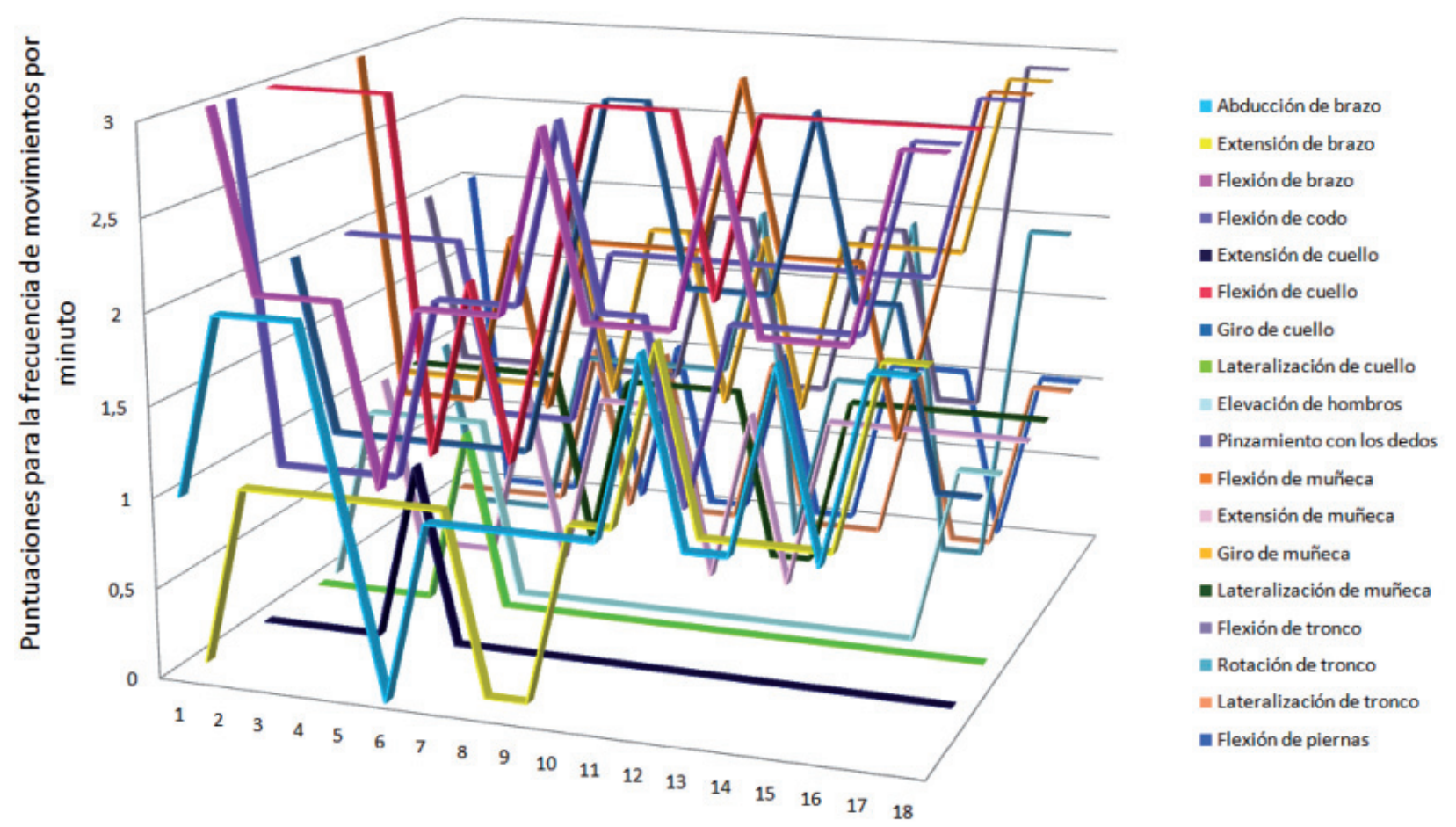

Figura 3: Variación en los niveles de riesgo para cada movimiento entre los puestos

Como resultado de la evaluación de la repetitividad de movimientos presente en los puestos se observó que ninguno suponía la exposición de los trabajadores a sobreesfuerzos.
Además, se constató que existía variedad en los niveles de riesgo asociados a los movimientos entre los puestos (Figura 3). Dicha variedad podía permitir alternar asignaciones que

\begin{tabular}{llllllllllllllllllll}
\hline Puesto & 1 & 2 & 3 & 4 & 5 & 6 & 7 & 8 & 9 & 10 & 11 & 12 & 13 & 14 & 15 & 16 & 17 & 18 \\
\hline Abducción de brazo & 1 & 2 & 2 & 2 & 1 & 0 & 1 & 1 & 1 & 1 & 1 & 2 & 1 & 1 & 2 & 1 & 2 & 2 \\
Extensión de brazo & 0 & 1 & 1 & 1 & 1 & 1 & 1 & 0 & 0 & 1 & 1 & 2 & 1 & 1 & 1 & 1 & 2 & 2 \\
Flexión de brazo & 3 & 2 & 2 & 2 & 1 & 2 & 2 & 2 & 3 & 2 & 2 & 2 & 3 & 2 & 2 & 2 & 3 & 3 \\
Flexión de codo & 3 & 1 & 1 & 1 & 1 & 2 & 2 & 2 & 3 & 2 & 2 & 1 & 2 & 2 & 2 & 2 & 3 & 3 \\
Extensión de cuello & 0 & 0 & 0 & 0 & 1 & 0 & 0 & 0 & 0 & 0 & 0 & 0 & 0 & 0 & 0 & 0 & 0 & 0 \\
Flexión de cuello & 3 & 3 & 3 & 3 & 1 & 2 & 1 & 2 & 3 & 3 & 3 & 2 & 3 & 3 & 3 & 3 & 3 & 3 \\
Giro de cuello & 2 & 1 & 1 & 1 & 1 & 1 & 1 & 2 & 3 & 3 & 2 & 2 & 2 & 3 & 2 & 2 & 1 & 1 \\
Lateralización de cuello & 0 & 0 & 0 & 0 & 1 & 0 & 0 & 0 & 0 & 0 & 0 & 0 & 0 & 0 & 0 & 0 & 0 & 0 \\
Elevación de hombros & 0 & 1 & 1 & 1 & 1 & 0 & 0 & 0 & 0 & 0 & 0 & 0 & 0 & 0 & 0 & 0 & 1 & 1 \\
Pinzamiento con los & 2 & 2 & 2 & 2 & 1 & 1 & 1 & 2 & 2 & 2 & 2 & 2 & 2 & 2 & 2 & 2 & 3 & 3 \\
dedos & 3 & 1 & 1 & 1 & 2 & 1 & 2 & 2 & 2 & 2 & 3 & 2 & 2 & 2 & 1 & 2 & 3 & 3 \\
Flexión de muñeca & 1 & 0 & 0 & 0 & 1 & 0 & 1 & 1 & 1 & 0 & 1 & 0 & 1 & 1 & 1 & 1 & 1 & 1 \\
Extensión de muñeca & 1 & 1 & 1 & 1 & 1 & 2 & 1 & 2 & 2 & 1 & 2 & 1 & 2 & 2 & 2 & 2 & 3 & 3 \\
Giro de muñeca & 1 & 1 & 1 & 1 & 1 & 0 & 1 & 1 & 1 & 1 & 0 & 0 & 1 & 1 & 1 & 1 & 1 & 1 \\
Lateralización de & 2 & 1 & 1 & 1 & 2 & 1 & 1 & 1 & 2 & 2 & 1 & 1 & 2 & 2 & 1 & 1 & 3 & 3 \\
muñeca & 0 & 0 & 0 & 0 & 0 & 0 & 0 & 0 & 0 & 0 & 0 & 0 & 0 & 0 & 0 & 0 & 0 & 0 \\
Flexión de tronco & 1 & 0 & 0 & 0 & 1 & 1 & 1 & 1 & 1 & 2 & 0 & 1 & 1 & 2 & 0 & 0 & 2 & 2 \\
Extensión de tronco & 0 & 0 & 0 & 0 & 1 & 0 & 1 & 0 & 0 & 1 & 0 & 0 & 0 & 1 & 0 & 0 & 1 & 1 \\
Rotación de tronco & 2 & 0 & 0 & 0 & 1 & 0 & 1 & 0 & 0 & 1 & 0 & 0 & 1 & 1 & 1 & 0 & 1 & 1
\end{tabular}

Tabla 1: Puntuaciones de los ítems de movimiento de los 18 puestos analizados 
supusieran acumulación de fatiga en un grupo muscular con otras que favorecieran la recuperación de éste. En los casos con menor variedad en los niveles de riesgo, éstos eran bajos y, por lo tanto, asignaciones consecutivas entre dichos puestos no resultarían, según el equipo médico de la empresa, perjudiciales.

\section{PERFILES COMPETENCIALES Y PARÁMETROS DE DPI-A}

Para el caso en estudio se obtuvieron los perfiles competenciales de los puestos recogidos en las Tablas 1 y 2.

\begin{tabular}{llll} 
Trabajador & 12 & 14 & 15 \\
Extensión de cuello & 0 & 2 & 0 \\
Flexión de cuello & 0 & 1 & 0 \\
Giro de cuello & 0 & 1 & 0 \\
Lateralización de cuello & 0 & 2 & 0 \\
Elevación de hombros & 2 & 1 & 0 \\
Flexión de tronco & 2 & 1 & 0 \\
Rotación de tronco & 3 & 2 & 0 \\
Lateralización de tronco & 3 & 1 & 0 \\
Flexión de piernas & 2 & 0 & 2 \\
\hline
\end{tabular}

Tabla 3: Trabajadores con limitaciones en ciertos movimientos

\begin{tabular}{|c|c|c|c|c|c|c|c|c|c|c|c|c|c|c|c|c|c|c|}
\hline Puesto & 1 & 2 & 3 & 4 & 5 & 6 & 7 & 8 & 9 & 10 & 11 & 12 & 13 & 14 & 15 & 16 & 17 & 18 \\
\hline Estar de pie & $\bullet$ & & & & & $\bullet$ & $\bullet$ & $\bullet$ & $\bullet$ & $\bullet$ & & $\bullet$ & $\bullet$ & & $\bullet$ & $\bullet$ & $\bullet$ & $\bullet$ \\
\hline $\begin{array}{l}\text { Subir (escaleras, } \\
\text { pendientes) }\end{array}$ & $\bullet$ & & & & & $\bullet$ & & & & & & & & & & & & \\
\hline $\begin{array}{l}\text { Trepar (con piernas y } \\
\text { brazos) }\end{array}$ & & & & & & $\bullet$ & & & & & & & & & & & & \\
\hline Coordinar movimientos & $\bullet$ & & & & $\bullet$ & $\bullet$ & $\bullet$ & $\bullet$ & $\bullet$ & $\bullet$ & & & $\bullet$ & & $\bullet$ & $\bullet$ & $\bullet$ & $\bullet$ \\
\hline $\begin{array}{l}\text { Ejercer fuerza estando } \\
\text { quieto }\end{array}$ & $\bullet$ & & & & $\bullet$ & $\bullet$ & $\bullet$ & $\bullet$ & $\bullet$ & $\bullet$ & & $\bullet$ & $\bullet$ & & & $\bullet$ & $\bullet$ & $\bullet$ \\
\hline $\begin{array}{l}\text { Ejercer fuerza en } \\
\text { movimiento }\end{array}$ & $\bullet$ & & & & & $\bullet$ & & & & & & & & & & & & \\
\hline Conducir vehículos & & & & & $\bullet$ & & & & & & & & & & & & & \\
\hline Trabajar en las Alturas & & & & & & $\bullet$ & & & & & & & & & & & & \\
\hline $\begin{array}{l}\text { Razonar/Tomar decisiones } \\
\text { complejas }\end{array}$ & $\bullet$ & • & $\bullet$ & $\bullet$ & $\bullet$ & & & & & & $\bullet$ & & & & & $\bullet$ & & $\bullet$ \\
\hline Responsabilidad & $\bullet$ & $\bullet$ & $\bullet$ & $\bullet$ & $\bullet$ & $\bullet$ & & & & & $\bullet$ & & & & & $\bullet$ & $\bullet$ & $\bullet$ \\
\hline Iniciativa/Autonomía & & $\bullet$ & $\bullet$ & $\bullet$ & & & & & & & $\bullet$ & & & & & $\bullet$ & $\bullet$ & $\bullet$ \\
\hline Ver de lejos & $\bullet$ & & & & $\bullet$ & $\bullet$ & $\bullet$ & $\bullet$ & $\bullet$ & $\bullet$ & & & & & & & $\bullet$ & $\bullet$ \\
\hline Oír & $\bullet$ & & & & $\bullet$ & $\bullet$ & $\bullet$ & $\bullet$ & $\bullet$ & $\bullet$ & $\bullet$ & $\bullet$ & $\bullet$ & $\bullet$ & $\bullet$ & $\bullet$ & $\bullet$ & $\bullet$ \\
\hline $\begin{array}{l}\text { Localizar la Dirección del } \\
\text { sonido }\end{array}$ & $\bullet$ & & & & $\bullet$ & $\bullet$ & & & & & & & & & & & & $\bullet$ \\
\hline Escribir & & $\bullet$ & $\bullet$ & $\bullet$ & & & $\bullet$ & & & $\bullet$ & & & & & & $\bullet$ & & \\
\hline Usar el teclado & & $\bullet$ & $\bullet$ & $\bullet$ & & & & & & & & & & & & & & \\
\hline Usar el ratón & & $\bullet$ & $\bullet$ & $\bullet$ & & & & & & & & & & & & & & \\
\hline
\end{tabular}

Tabla 2: Capacidades necesarias en los 18 puestos analizados. Las celdas vacías indican que la capacidad no era necesaria en el puesto

También se obtuvieron los perfiles competenciales de los trabajadores. Para ello, el equipo médico determinó el grado de limitación de los trabajadores para realizar ciertos movimientos. Sólo en tres de ellos observaron limitaciones (Tabla 3). Respecto a las capacidades se registraron limitaciones únicamente en 4 trabajadores.

\begin{tabular}{lllll}
\hline Trabajador & 12 & 13 & 14 & 15 \\
\hline Estar de pie & $\bullet$ & & & \\
Subir (escaleras, pendientes) & $\bullet$ & & $\bullet$ & $\bullet$ \\
Trepar (con piernas y brazos) & $\bullet$ & & $\bullet$ & $\bullet$ \\
Coordinar movimientos & $\bullet$ & & & \\
Ejercer fuerza estando quieto & $\bullet$ & & $\bullet$ & \\
Ejercer fuerza en movimiento & $\bullet$ & & $\bullet$ & $\bullet$ \\
Conducir vehículos & $\bullet$ & $\bullet$ & $\bullet$ & \\
Trabajar en las Alturas & $\bullet$ & & $\bullet$ & $\bullet$ \\
\hline Ver de lejos & & $\bullet$ &
\end{tabular}

Tabla 4: Trabajadores con capacidades limitadas 
Seguidamente se recopilaron el conjunto de las asignaciones no deseadas. Dichas restricciones se obtuvieron del cruce de la información sobre las limitaciones de movimiento (Tabla 3 ) y en las capacidades de los trabajadores (Tabla 4), con la recopilada acerca de los movimientos (Tabla 1) y las capacidades requeridas en los puestos (Tabla 2). inclusión de los trabajadores con discapacidades no suponía que el algoritmo propusiera agendas de rotación con aptitudes significativamente peores que, por lo tanto, desaconsejaran la opción de la integración. Los experimentos se realizaron con el software MORE.

El algoritmo DPI-A fue ejecutado 10 veces para el

\begin{tabular}{lllllllllllllllllll}
\hline Puesto & 1 & 2 & 3 & 4 & 5 & 6 & 7 & 8 & 9 & 10 & 11 & 12 & 13 & 14 & 15 & 16 & 17 & 18 \\
\hline Trabajador 12 & $\bullet$ & & & & $\bullet$ & $\bullet$ & $\bullet$ & $\bullet$ & $\bullet$ & $\bullet$ & & $\bullet$ & $\bullet$ & & $\bullet$ & $\bullet$ & $\bullet$ & $\bullet$ \\
Trabajador 13 & $\bullet$ & & & & $\bullet$ & $\bullet$ & $\bullet$ & & $\bullet$ & $\bullet$ & & & & & & & $\bullet$ & $\bullet$ \\
Trabajador 14 & $\bullet$ & & & & $\bullet$ & $\bullet$ & $\bullet$ & $\bullet$ & $\bullet$ & $\bullet$ & & $\bullet$ & $\bullet$ & & & & $\bullet$ & $\bullet$ \\
Trabajador 15 & $\bullet$ & & & & & $\bullet$ & & & & & & & & & & & &
\end{tabular}

Tabla 5: Asignaciones vetadas trabajador-puesto (las celdas con punto indican que el trabajador no debe ser asignado al puesto)

\begin{tabular}{lll}
\hline Parámetro & Definición & Valores \\
\hline$n_{\text {wor }}$ & Número de trabajadores y puestos & 18 \\
$n_{\text {rot }}$ & Número de rotaciones & 4 \\
$n_{i t}$ & Número de ítems considerados en la fórmula de evaluación & 18 \\
$\mathrm{C}_{1} \ldots \mathrm{C}_{18}$ & Coeficientes de ponderación de los ítems & 1 \\
$\mathrm{f}_{\text {red }}$ & Factor de reducción & 3 \\
$\mathrm{tre}$ & Valor umbral & 1 \\
$\mathrm{t}_{1}$ & Duración de la Rotación 1 & 2 horas \\
$\mathrm{t}_{2}$ & Duración de la Rotación 2 & 2 horas \\
$\mathrm{t}_{3}$ & Duración de la Rotación 3 & 2 horas \\
$\mathrm{t}_{4}$ & Duración de la Rotación 4 & 1 hora \\
$\mathrm{p}_{1}$ & Duración de la pausa 1 (almuerzo) & 2 horas \\
$\mathrm{t}_{\text {max }}$ & Tiempo máximo consecutivo de permanencia en puestos similares & 2 horas \\
Gen & Número de generaciones tras las que se detendrá el algoritmo & 4000 \\
$n_{i}$ & Individuos en cada generación & 50 \\
$\mathrm{p}_{c}$ & Probabilidad de cruce & 0,6 \\
$\mathrm{p}_{\mathrm{m}}$ & Probabilidad de mutación & 0,3 \\
\hline
\end{tabular}

Tabla 6: Parámetros de ejecución de DPI-A

Finalmente se establecieron los valores de los parámetros necesarios para la ejecución de DPI-A (Tabla 6) mediante el software MORE. Dichos parámetros incluyen valores propios de los algoritmos genéticos y valores específicos empelados por DPI-A dependientes del caso de estudio.

\section{RESULTADOS}

Para la realización de los experimentos se definieron dos escenarios, el primero incluía a los trabajadores con discapacidades (12, 14 y 15), en adelante "Rotación con Integración" (RCI), y el segundo suponía que todos los trabajadores eran capaces de realizar todos los movimientos sin limitación y tampoco presentaban dificultades en ninguna capacidad, escenario identificado como "Rotación Sin Integración" (RSI). Se trataba con ello de demostrar que la escenario RCI con los parámetros indicados en (Tabla 6), y otras 10 para el escenario RSI con los mismo parámetros. El tiempo empleado por el algoritmo fue de 2 horas 47 minutos para RCI y de 1 hora 34 minutos para RSI, siendo mayor para RCI debido al mayor número de restricciones del problema. Ambos datos se consideraron tiempos de cálculo aceptables en el entorno del problema planteado. En todas las ejecuciones el algoritmo fue capaz de obtener soluciones factibles, y por lo tanto, sujetas a las restricciones del problema. El algoritmo DPI-A trata de minimizar el valor de la función objetivo (E), por lo que un valor menor de $\mathrm{E}$ supone una mejor asignación de los trabajadores. La mejor aptitud alcanzada en RCI fue de 607,11 (Tabla 6) mientras que RSI logró una aptitud de 567,85 (Tabla 7).

El estudio de la evolución de los valores de los ítems de movimiento a lo largo de las rotaciones para la mejor solución de RCI (Tabla 7) permitió comprobar la eficacia del algoritmo DPI-A para encontrar asignaciones capaces de 


\begin{tabular}{|c|c|c|c|c|c|}
\hline Trabajador & Rotación 1 & Rotación 2 & Rotación 3 & Rotación 4 & Coste Asignación \\
\hline 1 & Puesto 17 & Puesto 16 & Puesto 15 & Puesto 5 & 41,11 \\
\hline 2 & Puesto 14 & Puesto 3 & Puesto 1 & Puesto 6 & 32,75 \\
\hline 3 & Puesto 4 & Puesto 14 & Puesto 13 & Puesto 7 & 30,41 \\
\hline 4 & Puesto 1 & Puesto 6 & Puesto 14 & Puesto 4 & 31,62 \\
\hline 5 & Puesto 12 & Puesto 1 & Puesto 6 & Puesto 10 & 29,77 \\
\hline 6 & Puesto 7 & Puesto 18 & Puesto 2 & Puesto 9 & 28,59 \\
\hline 7 & Puesto 6 & Puesto 10 & Puesto 3 & Puesto 17 & 25,96 \\
\hline 8 & Puesto 8 & Puesto 13 & Puesto 12 & Puesto 8 & 34,11 \\
\hline 9 & Puesto 15 & Puesto 7 & Puesto 18 & Puesto 16 & 31,4 \\
\hline 10 & Puesto 18 & Puesto 8 & Puesto 10 & Puesto 2 & 43,42 \\
\hline 11 & Puesto 3 & Puesto 9 & Puesto 7 & Puesto 14 & 25,08 \\
\hline 12 & Puesto 11 & Puesto 4 & Puesto 11 & Puesto 3 & 42,04 \\
\hline 13 & Puesto 13 & Puesto 12 & Puesto 8 & Puesto 13 & 35,21 \\
\hline 14 & Puesto 16 & Puesto 2 & Puesto 16 & Puesto 11 & 55,71 \\
\hline 15 & Puesto 2 & Puesto 11 & Puesto 9 & Puesto 12 & 32,18 \\
\hline 16 & Puesto 9 & Puesto 5 & Puesto 17 & Puesto 15 & 33,95 \\
\hline 17 & Puesto 5 & Puesto 17 & Puesto 4 & Puesto 1 & 25,04 \\
\hline \multirow[t]{4}{*}{18} & Puesto 10 & Puesto 15 & Puesto 5 & Puesto 18 & 28,75 \\
\hline & & & \multicolumn{2}{|c|}{ Coste medio } & 33,32 \\
\hline & & & \multicolumn{2}{|c|}{ Desviación típicas } & 7,9 \\
\hline & & & \multicolumn{2}{|c|}{ Mejor Aptitud } & 607,1 \\
\hline
\end{tabular}

Tabla 7: Mejor solución encontrada en el escenario RCl en la ejecución 6

evitar el incremento progresivo de la mayoría de los ítems de movimiento, es decir, evitar la acumulación de la fatiga. La Figura 4 muestra la variación del valor de los ítems de movimiento para el trabajador 12 en la mejor solución alcanzada para RCI. Se observa que los ítems con valores iniciales superiores a 0 (elevación de hombros, flexión de tronco, giro de tronco, lateralización de tronco, flexión de piernas) mantienen el valor inicial. El algoritmo fue capaz de asignar al trabajador 12 a puestos que no exigían dichos movimientos, o el valor de los ítems era inferior al valor del parámetro tres $(1,5)$, valor por debajo del cual el equipo médico consideró que el puesto no provocaba fatiga. Además, los puestos asignados requerían capacidades para las que el trabajador no estaba limitado. El trabajador 14 fue asignado a puestos que aumentaron únicamente los ítems de flexión y rotación de cuello. Su asignación a puestos con estos movimientos de cuello no pudo ser evitada debido a que estaban presentes en todos ellos, provocando el incremento progresivo de los correspondientes ítems. Sólo se registró disminución después de la pausa para el almuerzo (Figura 5). Sin embargo, sí fue posible asignar al trabajador puestos que no exigían lateralización de cuello, elevación de hombros o movimientos del tronco, movimientos todos ellos para los que también estaba limitado. El equipo médico determinó que, a pesar de los incrementos progresivos registrados en los ítems de movimiento referidos, estos podían considerarse, en principio, aptos para el trabajador, a la espera controles médicos posteriores a la implantación de la agenda de rotación. Finalmente, el trabajador 15 fue asignado a puestos que no exigían la flexión de piernas que era el único movimiento para el que presentaba problemas, y por lo tanto, el ítem correspondiente se mantuvo constante al valor inicial de 2 . Todos los puestos asignados al trabajador 15 (2, 11, 9 y 12) eran compatibles con sus limitaciones (subir escaleras o pendientes, ejercer fuerza parado o caminando y trabajar en altura).

Sólo en el caso de los trabajadores 12 y 14, ambos con limitaciones en movimientos y capacidades, se repetía la asignación de un mismo puesto a lo largo de la jornada, pero nunca en rotaciones consecutivas. El resto de los trabajadores ocupaban puestos distintos en cada rotación.

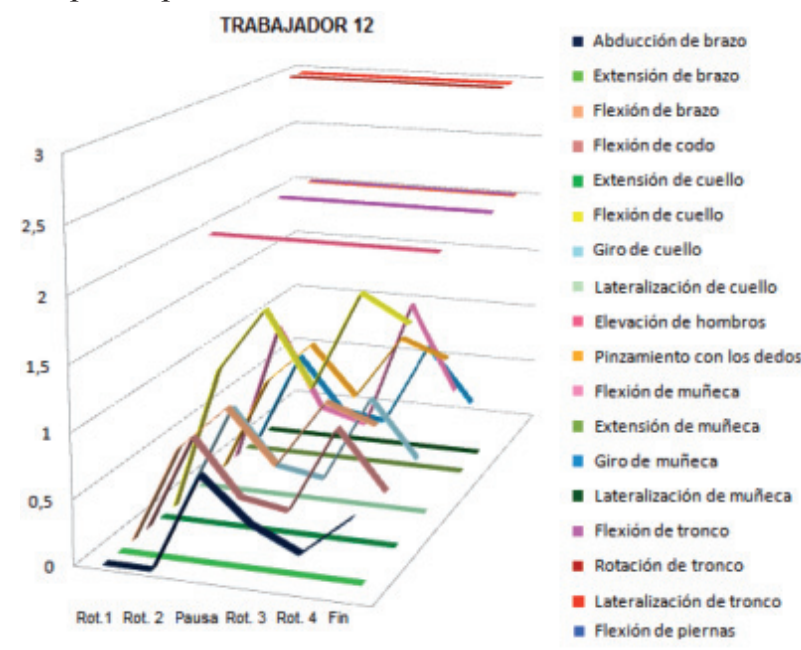

Figura 4: Variación de los valores de los ítems de movimiento del trabajador 12 


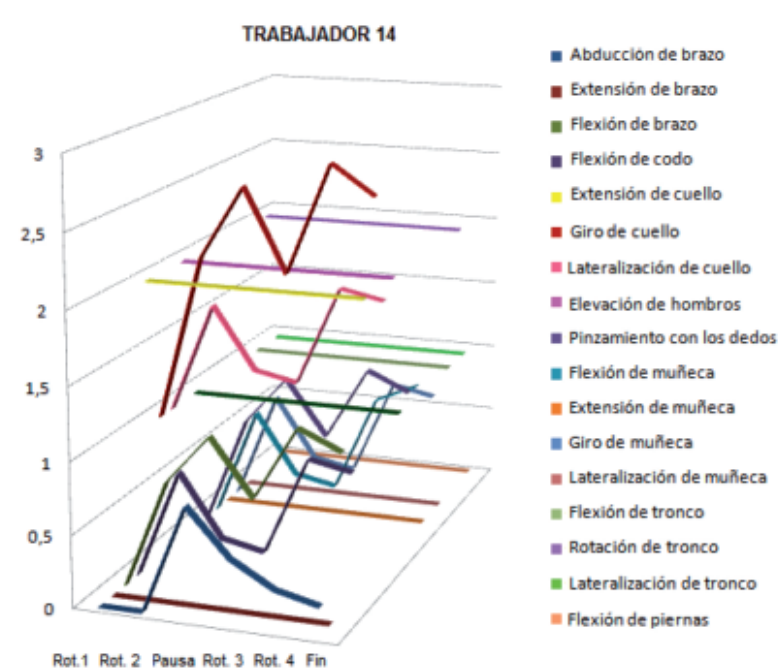

Figura 5: Variación de los valores de los ítems de movimiento del trabajador 74

\section{CONCLUSIONES}

La RPT es conocida como técnica organizativa que permite obtener diversos beneficios tanto para los trabajadores implicados como para la empresa implantadora. Los resultados de este trabajo han permitido comprobar que, además de lograr la disminución de la monotonía y prevenir la aparición de TMEs, la RPT permite la incorporación de trabajadores con discapacidad en los procesos de trabajo ordinarios, sin la necesidad de adaptaciones especiales de los entornos productivos. De esta forma se logra una integración mayor sin que esto suponga un deterioro de la producción y de las condiciones laborales del resto de trabajadores.

La inclusión de trabajadores con discapacidades no impide al algoritmo DPI-A lograr soluciones con variación en los puestos ocupados por el resto de trabajadores, contribuyendo a disminuir la monotonía y favoreciendo la alternancia de los grupos musculares empleados por estos.

\begin{tabular}{|c|c|c|c|c|c|}
\hline Trabajador & Rotación 1 & Rotación 2 & Rotación 3 & Rotación 4 & Coste Asignación \\
\hline 1 & Puesto 11 & Puesto 3 & Puesto 9 & Puesto 5 & 29,84 \\
\hline 2 & Puesto 5 & Puesto 15 & Puesto 13 & Puesto 12 & 20,18 \\
\hline 3 & Puesto 17 & Puesto 8 & Puesto 3 & Puesto 14 & 39,71 \\
\hline 4 & Puesto 13 & Puesto 7 & Puesto 14 & Puesto 2 & 28,46 \\
\hline 5 & Puesto 16 & Puesto 5 & Puesto 18 & Puesto 16 & 28,96 \\
\hline 6 & Puesto 14 & Puesto 4 & Puesto 1 & Puesto 6 & 32,75 \\
\hline 7 & Puesto 7 & Puesto 14 & Puesto 2 & Puesto 11 & 21,42 \\
\hline 8 & Puesto 12 & Puesto 1 & Puesto 6 & Puesto 10 & 29,77 \\
\hline 9 & Puesto 6 & Puesto 10 & Puesto 11 & Puesto 3 & 27 \\
\hline 12 & Puesto 18 & Puesto 6 & Puesto 10 & Puesto 15 & 38,36 \\
\hline 13 & Puesto 1 & Puesto 12 & Puesto 8 & Puesto 4 & 32,46 \\
\hline 14 & Puesto 8 & Puesto 13 & Puesto 12 & Puesto 9 & 36,77 \\
\hline 15 & Puesto 10 & Puesto 16 & Puesto 4 & Puesto 18 & 35,75 \\
\hline 16 & Puesto 4 & Puesto 18 & Puesto 16 & Puesto 7 & 33,38 \\
\hline 17 & Puesto 2 & Puesto 9 & Puesto 7 & Puesto 17 & 27,32 \\
\hline \multirow[t]{4}{*}{18} & Puesto 15 & Puesto 11 & Puesto 5 & Puesto 13 & 26,96 \\
\hline & & & \multicolumn{2}{|c|}{ Coste medio } & 32,36 \\
\hline & & & \multicolumn{2}{|c|}{ Desviación típicas } & 5,54 \\
\hline & & & \multicolumn{2}{|c|}{ Mejor Aptitud } & 567,84 \\
\hline
\end{tabular}

Tabla 8: Mejor solución encontrada en el escenario RSI en la ejecución 4

En relación al equilibrio de las asignaciones entre los trabajadores en las mejores soluciones de RCI y RSI, se observó que las asignaciones del escenario RSI eran más equilibradas que las de RCI, con una desviación típica de los coste de asignación de 7,9 en RCI y de 5,54 en RSI. Los desequilibrios mayores en RCI se debieron, por una parte, a los valores iniciales mayores a cero de ciertos ítems de movimiento de los trabajadores discapacitados, y por otra, a la rigidez impuesta por las restricciones derivadas de las discapacidades de los trabajadores, que disminuyeron la capacidad del algoritmo para lograr asignaciones con menor desequilibrio.
Además, tampoco supone que puestos considerados "ligeros" sean asignados a un único trabajador, con el consiguiente rechazo del resto de trabajadores. En el caso de estudio sólo el puesto 11 , de carácter administrativo, fue asignado únicamente a trabajadores con discapacidades, si bien su asignación se alternó entre los tres existentes. Aunque en el escenario RSI el algoritmo obtuvo una solución con una aptitud algo menor que la alcanzada para RCI, y con una uniformidad algo menor en la carga de los trabajadores, dicho pequeño empeoramiento puede considerarse sobradamente compensado por los beneficios socio-económicos derivados de la integración de trabajadores con discapacidades. 
No obstante, queda pendiente validar el resultado postimplantación de las agendas de rotación propuestas por el algoritmo DPI-A con integración de discapacitados. Para ello será necesaria la recopilación de datos pasado un tiempo suficiente tras su implantación. Dichos datos deberán ser comparados con las incidencias músculoesqueléticas anteriores a la propuesta adoptada. Debido al carácter acumulativo de los TMEs será necesario un tiempo suficientemente largo de observación; por lo menos de 2 años. Este plazo ha transcurrido ya en una línea de montaje de componentes de automóviles en la que se empleó el algoritmo DPI-A para establecer el plan de rotaciones bajo condiciones similares a las del caso de estudio planteado. En ese año, 2007, se registraron en dicha línea 186 asistencias médicas por TMEs. Tras la implantación de rotaciones, en 2008, el número de asistencias médicas se redujo a 169, y en 2009 a 112, lográndose una reducción del 40\% aproximadamente en la incidencia de los TMEs, sin que la consideración de las discapacidades supusiera una merma en la calidad de la solución implantada.

\section{AGRADECIMIENTOS}

Agradecemos a la Universidad Politécnica de Valencia su apoyo a esta investigación a través de su Programa de Apoyo a la Investigación y Desarrollo 2009 y su financiación a través de los proyectos PAID-06-09/2902 y PAID-05-09/4215.

\section{BIBLIOGRAFÍA}

- "Campaña europea contra los trastornos musculoesqueléticos". DYNA Ingeniería e Industria. 2010. Vol.826 p.336

- "Encuesta de Discapacidad, Autonomía personal y situaciones de Dependencia". Instituto Nacional de Estadística. España. 2008.

- Armengou-Foix R. La responsabilidad social corporativa: Protección de trabajadores sensibles a determinados riesgos [en línea]. Asepeyo, [ref. de Julio de 2010]. Disponible en Web: http://iwebintra.asepeyo. es/Web/Biblioteca.nsf/ficheros/monografia28abril2006. pdf/\$file/monografia28abril2006.pdf

- Bernard B. "Musculoskeletal disorders and workplace factors: A critical review of epidemiological evidence for work-related musculoskeletal disorders of the neck, upper extremity, and low back". National Institute for Occupational Safety and Health (NIOSH). 1997.

- Carnahan BJ, Redfern MS, Norman B. "Designing safe Job Rotations chedules using optimization and heuristic search". Ergonomics. 2000. Vol. 43 p.543-560.

- Costa AM, Miralles C. "Job rotation in assembly lines employing disabled workers". Int. J. Production Economics. 2009. Vol.120 p.625 - 632.
- Diego-Mas JA, Asensio-Cuesta S, Sanchez-Romero MA, Artacho-Ramirez MA "A multi-criteria genetic algorithm for the generation of Job Rotations chedules". International Journal of Industrial Ergonomics. 2008. Vol. 39 p.23-33.

- Diego-Mas JA, Asensio-Cuesta S. Rotación de puestos de trabajo: Método MORE. [en línea]. Universidad Politécnica e Valencia, [ref. de Julio de 2010]. Disponible en Web: <http://www.ergonautas.upv.es/ metodos/more/more.htm>.

- España. Ley orgánica 13/1982, de 7 de abril, de Integración Social de los Minusválidos. Boletín Oficial del Estado, 30 de Abril de 1982, núm. 103, p. 11106 a 11112.

- Goldberg DE. Genetic Algorithms in Search, Optimization and Machine Learning. Addison-Wesley Publishing Company Inc. Massachusetts. 1989. p.412. ISBN: 0-201-15767-5.

- López-Lasaola R. "¿Es rentable la prevencion?". DYNA Ingeniería e Industria. 2008. Vol.83-2 p.119-122

- Millas V, Calderón B. "Guía Práctica para la elaboración de un programa de integración laboral de personas con discapacidad". Acción RSE. 2005.

- Oromí A. El 80\% de las empresas españolas no cumple la ley de integración de discapacitados [en línea]. Fundación prevent. [ref. de Octubre de 2010]. Disponible en Web: http://www.fundacionprevent.com/ Fundacion/pdf/45.pdf.

- Papadimitriou CH, Steiglitz K., Combinatorial Optimization: Algorithms and Complexity. Dover Publications Inc. New York. 1982. ISBN 0-486-402584.

- Seckiner SU y Kurt M. 2007. "Ant colony optimization for the Job Rotation scheduling problem". Applied Mathematics and Computation. 2007. Vol. 201 p.149160.

- Tharmmaphornphilas W, Norman B. "A quantitative method for determining proper Job Rotation intervals". Annals of operations research. 2004. Vol. 128 p.251266.

- Vézina N. "Rotation implantation: what is at stake? What are the markers". WorkShop Principles and Practices of Job Rotation". Centre of Research Expertise for the Prevention of Musculoskeletal Disorders. 2004. p.1-22. 